\title{
Eggert's Conjecture for 2-Generated Nilpotent Algebras
}

\section{Miroslav Korbelar*}

Charles University, Faculty of Mathematics and Physics, Department of Algebra, Sokolovska, Czech Republic

\begin{abstract}
Let $A$ be a commutative nilpotent finitely-dimensional algebra over a field $F$ of characteristic $p>0$. A conjecture of Eggert says that $p \cdot \operatorname{dim} A^{(p)} \operatorname{dim} \mathrm{A}$, where $A^{(p)}$ is the subalgebra of $A$ generated by elements $a^{p}, a \in A$. We show that the conjecture holds if $A^{(p)}$ is at most 2-generated.
\end{abstract}

Keywords: Nilpotent algebra; Eggert's conjecture; Commutative nilpotent ring; Polynomial bases

\section{Introduction}

Let $F$ be a field of characteristic $p>0$ and $A$ a commutative (associative) nilpotent finite-dimensional algebra over $F$. Let $A^{(p)}$ be the subalgebra generated by the set $\left\{a^{p} \mid a \in A\right\}$. N. Eggert [1] conjectured that

$$
p \cdot \operatorname{dim} A^{(p)} \leq \operatorname{dim} A .
$$

This conjecture gives an answer to the problem, when a finite abelian group is isomorphic to the adjoint group of some finite commutative nilpotent $F$-algebra. Recall that the adjoint group of $A$ is the set $A$ with the operation $x \circ y=x+y+x y$ for every $x, y \in A$.

Validity of this hypothesis would also have influence on an estimation of a (Prüfer) rank of a product of two (abelian) p-groups.

N. Eggert proved his conjecture only when $\operatorname{dim} A^{(p)} \leq 2$. Five years later, R. Bautista [2] proved it when $\operatorname{dim} A^{(p)}=3$. C. Stack confirmed this results in Stack et al. [3,4], but provided shorter proofs. Finally, Amberg and Kazarin [5] proved the conjecture for the case $\operatorname{dim} A^{(p)} \leq 4$.

Another type of results presented by McLean [6,7]. He showed that this conjecture is true if the algebra $A$ is either radical of a group algebra of a finite abelian group or $A$ is graded and at least one of the following conditions is fulfilled:

(i) $\quad p=2$ and $\left(A^{(p)}\right)^{4}=0$.

(ii) $A^{(p)}$ is 2-generated.

(iii) $\left(A^{(p)}\right)^{3}=0$.

(iv) $n<3 p$ and $3 \leq \mathrm{s}-1 \leq p$, where $n$ is the number of generators of $A^{(p)}$ and $s$ is the index of nilpotence of $A^{(p)}$.

We also should mention the result of Gorlov [8]. He proved the conjecture for nilpotent algebras $A$ with a metacyclic adjoint group.

One paper concerning Eggert's conjecture appeared in 2002 and the author L. Hammoudi [9] claimed he proved it. But, as Amberg [10] and McLean [7] have shown, his proof was incorrect.

In this short note we sketch out the main steps of the proof that Eggert's conjecture is true if the subalgebra $A^{(p)}$ has at most two generators. For the details, the reader is referred to Korbelar [11].

Since we will deal with nilpotency and commutativity only, we point out that the word 'algebra' will mean a commutative one and not necessary possesing a unit.

For an algebra $A$ and a subset $X \subseteq A$ we denote $\langle X\rangle$ ([X], resp.) the algebra (vector space, resp.) generated by $X$.
An algebra $A$ is called nilpotent if $A^{m}=0$ for some $m \in \mathrm{N}$.

Through this paper let always $F$ be a field of characteristic $p>0$ and $R=F[x, y]$ be the ring of polynomials over the variables $x, y$ and the field $F$.

We start with the remark, that the number of any minimal generating set of a finite generated nilpotent $F$-algebra $A$ is equal to $\operatorname{dim} A / A^{2}$. This implies the following:

Lemma 1.1. Suppose that Eggert's conjecture holds for every nilpotent 2-generated $F$-algebra. Then it also holds for every nilpotent $F$ -algebra $A$ such that $A(p)$ is a 2-generated $F$-algebra.

In the rest we deal with 2-generated nilpotent algebras.

\section{Bases of Nilpotent Algebras}

We will use the well-known concept of monomial ordering and standard bases.

$$
\text { For } \alpha=(i, j) \in \mathbb{N}_{0}^{2} \text { put }
$$$$
x^{\alpha}=x^{i} y^{j} \in F[x, y] \text {. }
$$

Denote $[X]_{0}=\left\{x^{\alpha} \mid \alpha \in \mathbb{N}_{0}^{2}\right\} \cup\{0\}$ the multiplicative monoid with the lexicographical ordering $\leq$ such that

$$
x^{(i, j)} \leq x^{\left(i^{\prime}, j^{\prime}\right)} \Leftrightarrow i<i^{\prime} \vee\left(i=i^{\prime} \wedge j \leq j^{\prime}\right)
$$

and

$$
x^{(i, j)} \leq 0
$$

for every $(i, j),\left(i^{\prime}, j^{\prime}\right) \in \mathbb{N}_{0}^{2}$

$$
\text { For } 0 \neq f=\sum_{\alpha} \lambda_{\alpha} x^{\alpha} \in F[x, y] \text { put }
$$$$
\mathrm{m}(f)=\mid \min \left\{x^{\alpha} \mid \lambda_{\alpha} \neq 0\right\}
$$

$m(0)=0$.

Finally, $f$ will be called normal iff $\lambda_{a 0}=1$, where $\mathrm{m}(f)=\boldsymbol{x}^{\alpha 0}$, and $\mathrm{m}(f)$ $<\pi \boldsymbol{x}^{\alpha}$ implies $\lambda_{\alpha}=0$ for every

${ }^{*}$ Corresponding author: Miroslav Korbelar, Faculty of Mathematics and Physics, Department of Algebra, Charles University, Sokolovska 83, 18675 Prague 8, Czech Republic, Tel: +420 224491 111; E-mail: miroslav.korbelar@gmail.com

Received July 24, 2015; Accepted August 03, 2015; Published August 31, 2015

Citation: Korbelar M (2015) Eggert's Conjecture for 2-Generated Nilpotent Algebras. J Generalized Lie Theory Appl S1: 001. doi:10.4172/1736-4337.S1-001

Copyright: @ 2015 Korbelar M, et al. This is an open-access article distributed under the terms of the Creative Commons Attribution License, which permits unrestricted use, distribution, and reproduction in any medium, provided the original author and source are credited. 


$$
\alpha \in \mathbb{N}_{0}^{2}
$$

This function $\mathrm{m}: F[x, y] \rightarrow[X]_{0}$ has common properties of a valuation:

(i) $\mathrm{m}(f g)=\mathrm{m}(f) \mathrm{m}(g)$. $<\mathrm{m}(g)$.

(ii) $\mathrm{m}(f+g) \geq \min \{\mathrm{m}(f) ; \mathrm{m}(g) g$. Moreover, $\mathrm{m}(f+g)=\mathrm{m}(f)$ if $\mathrm{m}(f)$

(iii) $\mathrm{m}\left(f\left(x^{p}, y^{p}\right)\right)=\mathrm{m}(f)^{\mathrm{p}}$.

for every $f, g \in F[x, y]$.

Finally. a set $\mathcal{X} \subseteq\left\{x^{\alpha} \mid \alpha \in \mathbb{N}_{0}^{2}\right\}$ will be called upper (lower, resp.) if $\mathrm{x}^{\alpha} \in \mathcal{X}$ and $x^{\alpha} \mid x^{\beta}\left(x^{\beta} \mid x^{\alpha}\right.$, resp.) implies $x^{\beta} \in \mathcal{X}$ for every $x^{\alpha}, x \in[X]_{0}$.

Definition 2.1. Let $A$ be a nilpotent $F$-algebra generated by $\left\{a_{1}\right.$, $\left.a_{2}\right\}$. Put

$C_{A}\left(a_{1}, a_{2}\right)=\left\{u \in[X]_{0}(\exists f \in R x+R y) \mathrm{m}(f)=u \wedge f\left(a_{1}, a_{2}\right)=0\right\}$

and

$\mathcal{B}_{A}\left(a_{1}, a_{2}\right)=[X]_{0} \backslash \mathcal{C}_{A}\left(a_{1}, a_{2}\right)$.

Proposition 2.2. Let $A$ be a nilpotent $F$-algebra generated by $\left\{a_{1}\right.$, $\left.a_{2}\right\}$. Then:

(i) $\mathcal{C}_{\mathrm{A}}\left(a_{1}, a_{2}\right)$ is an upper set and $0 \in \mathcal{C}_{\mathrm{A}}\left(a_{1}, a_{2}\right)$.

(ii) $\mathcal{B}_{A}\left(a_{1}, a_{2}\right)$ is a lower set and $1 \in \mathcal{B}_{\mathrm{A}}\left(a_{1}, a_{2}\right)$.

(iii) The set $\left\{x^{\alpha}\left(a_{1}, a_{2}\right) \mid 1 \neq x^{\alpha} \in \mathcal{B}_{\mathrm{A}}\left(a_{1}, a_{2}\right)\right\}$ is a basis of $A$. In particular, $\mathcal{B}_{\mathrm{A}}\left(a_{1}, a_{2}\right)$ is finite.

(iv) $\mathcal{C}_{\mathrm{A}}\left(a_{1}, a_{2}\right)=\left\{u[X]_{0} \mid(\exists f \in R x+R y) \mathrm{m}(f)=u^{\wedge} f\left(a_{1}, a_{2}\right)=0 \wedge f\right.$ is normal $\{\{0\}$.

Definition 2.3. Let $A$ be a nilpotent $F$-algebra generated by $\left\{a_{1}\right.$, $\left.a_{2}\right\}$. Denote

$$
\begin{aligned}
& n_{0}=\#\left\{x^{\alpha} \in \mathcal{B}_{A}\left(a_{1}, a_{2}\right) \mid \alpha \in \mathbb{N}_{0} \times\{0\}-1,\right. \\
& d_{i}=\#\left\{x^{\alpha} \in \mathcal{B}_{A}\left(a_{1}, a_{2}\right) \alpha \in\{i\} \times \mathbb{N}_{0}\right\}, \\
& \overline{n_{0}}=\#\left\{x^{\alpha} \in \mathcal{B}_{A^{(p)}}\left(a_{1}^{p}, a_{2}^{p}\right) \alpha \in \mathbb{N}_{0} \times\{0\}-1,\right. \\
& \bar{d}_{i}=\# \mathrm{x}^{\alpha} \in \mathcal{B}_{A^{(p)}}\left(a_{1}^{p}, a_{2}^{p}\right) \alpha \in\{i\} \times \mathbb{N}_{0}
\end{aligned}
$$

and

$$
D_{i}=\sum_{k=p i}^{p i+p-1} d_{k}
$$

for $i \in \mathbb{N}_{0}$

Lemma 2.4. Let $A$ be a nilpotent $F$-algebra generated by $\left\{a_{1}, a_{2}\right\}$. Then:
(i) $\bar{d}++\bar{d}^{-}=\left|\quad\left(a_{1}^{p}, a_{2}^{p}\right)\right|=1+\operatorname{dim} A^{(~)}$.
(ii) $D_{0}+{ }^{-}=\left|\quad\left(a_{1}, a_{2}\right)\right|=1+\operatorname{dim} A$.
(iii) The set $\left\{x^{\alpha}\left(a_{1}^{p}, a_{2}^{p}\right) \mid 1 \neq x^{\alpha} \in \quad\left(a_{1}^{p}, a_{2}^{p}\right)\right\}$ is a basis of $A^{(\mathrm{p})}$

\section{Eggert's Conjecture for 2-generated Algebras}

Let $I \subseteq R x+R y$ be an ideal in $R$ such that $A=R x+R y / I$ is a nonzero nilpotent $F$-algebra.
We have $A=\langle x+I, y+I\rangle$ and $A^{(p)}=\left\langle x^{p}+I, y^{p}+I\right\rangle$.

By definition of $\mathcal{C}_{\mathrm{A}}(x+I ; y+I)$ there are $f_{\mathrm{i}} \in R x+R y, 0 \leq i \leq n_{0}+1$, such that $\mathrm{m}\left(\boldsymbol{f}_{\mathrm{i}}\right)=x^{(\mathrm{i}, d i)}, \boldsymbol{f}_{\mathrm{i}} \in \mathrm{I}$ and $\boldsymbol{f}_{\mathrm{i}}$ are normal.

The main idea of the proof lies in the fact that taking a normal polynomial from $I$, dividing it by $x$ and then multiplying by some suitable $y^{k}$, we get again a member of $I$ (3.3). Then, using binomial formula in a suitable way, we obtain a polynomial that will estimate the number $\bar{d}_{i} z$ (see 3.4 and the definition of $\mathcal{B}_{A^{(p)}}\left(a_{1}^{p}, a_{2}^{p}\right)$ )

Lemma 3.1. (i) $f_{0}=y^{d_{0}}-x h_{0}$, where $h_{0} \in R$, and $f_{n_{0}+1}=x^{n_{0}}$.

(ii) $x f_{i} \in R f_{i+1}+\cdots+R f_{n_{0}+1}$ for $i=0, \ldots, n_{0}$.

Definition 3.2. Denote

$w_{\mathrm{A}}=\max \mathcal{B}_{\mathrm{A}}(x+I, y+I)$.

For $0 \leq i \leq \overline{n_{0}}$ denote

$m_{i} \in \mathbb{N}_{0}$

the least integer such that $p i \leq m_{i} \leq p i+p-1$ and $d_{p i} \geq \ldots \geq d_{m i}=d_{m i+1}$ $=\ldots=d_{p i+p-1}$. Put

$$
l_{i}=\left(\sum_{k=p i}^{m_{i}-1}\left(d_{k}-1\right)\right)-(p-1) d_{m_{i}} .
$$

Following lemma is obtained using induction.

Lemma 3.3. Let $1 \leq i \leq n_{0}+1$ and $0 \neq f \in I$ be such that $\mathrm{m}(f) x^{i}$. Then $y_{\mathrm{i}-1}^{\mathrm{d}}{ }^{-1}(f / x)+\mathrm{I} \in\left[w_{\mathrm{A}}+I\right]$.

The proof of the next proposition uses only the binomial formula. It finds the particular polynomial the we need to make an estimation of the numbers $D_{i}$ and thus of the dimension of $A^{(p)}$.

\section{Proposition 3.4.}

(i) If $0 \leq i<\overline{n_{0}}$ and $l_{i} \geq 0$, then $y^{l_{i}} x^{p i}\left(f_{m_{i}} / x^{m_{i}}\right)^{p}+I \in\left[w_{A}+I\right]$

(ii) If $0 \leq i<\overline{n_{0}}$ and $l_{i}<0$, then $x^{p i}\left(f_{m_{i}} / x^{m_{i}}\right)^{p} \in I$

(iii) If $i=\overline{n_{0}}$, then $y^{D_{i}-1} x^{p i}+I \in\left[w_{A}+I\right]$.

Now, only exploring carefully the previous cases for $i$ and $l_{i}$ we get the following interesting claim. It says that the inequality " $p \bar{d}_{i} \leq D_{i}$ " holds for almost every $i$.

Theorem 3.5. One of the following cases takes place:

(i) $p \bar{d} \bar{n}_{0} \leq D_{\bar{n}_{0}}+p-2$ and $p \bar{d}_{i} \leq D_{i}+1$ for every $0 \leq i<\overline{n_{0}}$. Moreover,

$p \bar{d}_{i_{0}}=D_{i_{0}}+1$ for at most one $0 \leq i_{0}<\overline{n_{0}}$

(ii) $p \bar{d} \overline{n_{0}} \leq D_{n_{0}}+p-1$ and $p \bar{d} i \leq D_{i}$ for every $0 \leq i<\overline{n_{0}}$

And our main result is just an easy corollary of this and 1.1.

Theorem 3.6. Let $A$ be a nilpotent $F$-algebra, char $F=p>0$, such that $A^{(p)}$ is 2-generated. Then $p \cdot \operatorname{dim} A^{(p)} \operatorname{dim} A$.

\section{References}

1. Eggert N (1971) Quasi regular groups of finite commutative nilpotent algebras. Pacific J Math 36: 631-634.

2. Bautista R (1976) Units of finite algebras. An. Inst. Mat. Univ. Nac. Autonoma Mexico 16: 1-78. 
Citation: Korbelar M (2015) Eggert's Conjecture for 2-Generated Nilpotent Algebras. J Generalized Lie Theory Appl S1: 001. doi:10.4172/1736-4337. S1-001

Page 3 of 3

3. Stack C (1996) Dimensions of nilpotent algebras over fields of prime characteristic. Pacific J Math 176: 263-266.

4. Stack C (1998) Some results on the structure of finite nilpotent algebras over field of prime characteristic. J. Combin. Math. Combin Comput 28: 327-335.

5. Amberg B and Kazarin LS (2001) Commutative nilpotent $p$-algebras with small dimension. Quaderni di Mat. (Napoli) 8: 1-20.

6. McLean KR (2004) Eggert's conjecture on nilpotent algebras. Comm Algebra 32: $997-1006$.

7. McLean KR (2006) Graded nilpotent algebras and Eggert's conjecture. Comm Algebra 34: 4427- 4439 .
8. Gorlov VO (1995) Finite nilpotent algebras with metacyclic adjoint group Ukrain Math Z 47: 1426-1431.

9. Hammoudi L (2002) Eggert's conjecture on the dimensions of nilpotent algebras. Pacific J Math 202: 93-97.

10. Amberg B and Kazarin LS (2005) Nilpotent p-algebras and factorized p-groups. Proceedings of Groups St. Andrews 1: 130-147.

11. Korbelar M (2010) 2-generated nilpotent algebras and Eggert's conjecture. Journal of Algebra 324: 1558-1576. 